\title{
PREFACE: EXPERIMENTAL MULTIPHASE FLOW DYNAMICS
}

I am delighted to present this special issue of Experimental Multiphase Flow Dynamics related to the understanding of phenomena and industrial application for gas-liquid two-phase systems.

Multiphase flows are flow types of a mixed flow of two or more phases: gas-liquid, solidliquid, solid-gas, solid-gas-liquid, liquid-liquid (immiscible liquids). The flows are widely seen in various fields, such as boilers in both conventional and nuclear power plants, petroleum pipelines, heat exchanger systems, environmental systems, biological systems, the food industry, and most chemical processes. The understanding of the multiphase flow dynamics is essential for the optimal design and/or operation for such plants and systems. Due to the development of computational fluid dynamics in recent years, detailed analysis of multiphase flow phenomena has progressed. However, since the phenomenon of multiphase flow is very complicated, it is desired to verify the analysis code and the model incorporated in the code with appropriate experimental data. This Special Issue of "Experimental Multiphase Flow Dynamics" is devoted to recent advances in experimental measurements and/or techniques and new experimental data by Japanese researchers. Especially, papers related to the gas-liquid two-phase systems (microbubble, mist, bioreactor, heat pipe, etc.) were collected which address fundamental science as well as engineering applications.

As guest editor, I would like to thank all authors who contributed to this special issue and the Editors-in-Chief, who enabled me to assemble it.

\section{Guest Editor:}

Akimaro Kawahara

Advanced Thermal and Fluid Energy System

Division of Industrial Fundamentals

Faculty of Advanced Science and Technology

Kumamoto University

Japan 